\title{
Diagnóstico do gerenciamento de resíduos de serviços de saúde em estabelecimentos públicos de municípios que recebem Imposto sobre Circulação de Mercadorias e Serviços ecológico no Estado de Minas Gerais
}

Diagnostic management of medical waste in public establishments of municipalities that receive Ecological ICMS in the State of Minas Gerais

Tania Cristina Souza', Cristiane Frizzo de Oliveira ${ }^{2}$, Hiram Jackson Ferreira Sartori ${ }^{3}$

\begin{abstract}
RESUMO
O maior problema do descarte dos resíduos de serviço de saúde (RSS) é seu potencial de risco. Assim, o presente trabalho teve como objetivo avaliar a aplicação das diretrizes da Resolução CONAMA no 358/2005 em estabelecimentos públicos de serviços de saúde localizados em 48 municípios mineiros, contemplados pelo recebimento de Imposto sobre Circulação de Mercadorias e Serviços (ICMS) ecológico. O estudo foi realizado a partir da aplicação, nas Secretarias Municipais de Saúde das cidades pesquisadas, de um questionário sobre o gerenciamento dos RSS. No geral, foram observadas maiores dificuldades na fase intraestabelecimento, que envolve a elaboração e a implantação do Plano de Gerenciamento de Resíduos de Serviços de Saúde (PGRSS). A fase extraestabelecimento tem sido cumprida na maior parte dos municípios, provavelmente devido ao recebimento do ICMS ecológico, que inclui a destinação dos resíduos.
\end{abstract}

Palavras-chave: resíduos de serviços de saúde; estabelecimentos públicos de serviços de saúde; ICMS ecológico.

\begin{abstract}
The biggest problem of waste of health service (HCW) is their potential risks. Thus, this work has as objective the assess of applicability of CONAMA Resolution 358/2005 in the public healthcare establishments, located in 48 municipalities that receive Ecological ICMS in the state of Minas Gerais. The study was accomplished through a survey about healthcare waste management that was sent to the Municipal Healthcare Secretaries of the surveyed municipalities In general, greater difficulties in intra-establishment phase were observed, involving the development and implementation of PGRSS Extra-establishment phase, have been fulfilled in most municipalities, probably because of Ecological ICMS they received, including the disposal of waste.
\end{abstract}

Keywords: waste of health service; public healthcare establishments; ecological ICMS

\section{INTRODUÇÃO}

A aprovação da Lei n 12.305/2010 (BRASIL, 2010), que instituiu a Política Nacional de Resíduos Sólidos (PNRS), estabeleceu princípios, objetivos, diretrizes, metas e ações relacionadas ao gerenciamento dos resíduos sólidos no Brasil. Essa lei trouxe profundas mudanças nas relações sociais, políticas e econômicas, ao ressaltar, em um dos seus princípios, a responsabilidade compartilhada. Dessa maneira, todos os setores envolvidos no ciclo de vida do produto (geradores, consumidores/fabricantes, distribuidores, importadores e comerciantes) são responsáveis pelos resíduos gerados. Tudo indica que, nas próximas décadas, o gerenciamento de resíduos sólidos no Brasil sofrerá grandes transformações nesse sentido.

'Pós-graduanda em Gestão Ambiental de Resíduos Sólidos pelo Instituto de Educação Continuada (IEC) da Pontifícia Universidade Católica de Minas Gerais (PUC-Minas) Belo Horizonte (MG), Brasil.

${ }^{2}$ Graduanda em Engenharia Ambiental pela Universidade Federal de Minas Gerais (UFMG) - Belo Horizonte (MG), Brasil.

${ }^{3}$ Professor da PUC-Minas - Belo Horizonte (MG), Brasil.

Endereço para correspondência: Tania Cristina Souza - Rod. Prefeito Américo Gianetti, s/n, Edifício Minas, 10 Andar - Serra Verde - $30630-900$ - Belo Horizonte (MG), Brasil -

E-mail: tania.souza@meioambiente.mg.gov.br

Recebido: 31/03/14 - Aceito: 15/05/15 - Reg. ABES: 132781 
Até a década de 1980, todos os resíduos provenientes de hospitais eram considerados perigosos, cuja denominação era "lixo hospitalar" (BRASIL, 2001). Nos tempos atuais, esse termo foi substituído por resíduos de serviços de saúde (RSS), que engloba todos os tipos de estabelecimentos prestadores de assistência à saúde humana e animal hospitais, ambulatórios, consultórios médicos e odontológicos, laboratórios, farmácias, clínicas veterinárias, dentre outros.

Os procedimentos de classificação e gerenciamento dos RSS no Brasil são definidos em Normas da Associação Brasileira de Normas Técnicas (ABNT), NBR 10.004. (2004), e Regulamentos estabelecidos pelo Sistema Nacional do Meio Ambiente (SISNAMA), composto por Conselho Nacional do Meio Ambiente (CONAMA), Ministério do Meio Ambiente (MMA), Instituto Brasileiro do Meio Ambiente e dos Recursos Naturais Renováveis (IBAMA), dentre outros; e pelo Sistema Nacional de Vigilância Sanitária (SNVS), que abrange a Agência Nacional de Vigilância Sanitária (ANVISA) e outros órgãos.

Dentre as legislações no âmbito da gestão dos RSS, destacam-se a Resolução da Diretoria Colegiada (RDC) ANVISA n 306, de 7 de dezembro de 2004, e a Resolução CONAMA no 358, de 29 de abril de 2005, que estabelecem definições, classificação e procedimentos mínimos para o gerenciamento dos RSS. A Resolução no 358/2005 (BRASIL, 2005) dispõe sobre o regulamento técnico para o gerenciamento dos RSS na fase extraestabelecimento (tratamento e destinação final), buscando harmonizar os princípios de gerenciamento interno contemplados na RDC ANVISA nº 306/2004.

Os RSS são classificados conforme o seu grau de risco, de acordo com a Resolução CONAMA nº 358/2005, sendo:

- Grupo A: resíduos com a possível presença de agentes biológicos que, por suas características de maior virulência ou concentração, podem apresentar risco de infecção;

- Grupo B: resíduos contendo substâncias químicas que podem apresentar risco à saúde pública ou ao meio ambiente, dependendo de suas características de inflamabilidade, corrosividade, reatividade e toxicidade;

- Grupo C: quaisquer materiais resultantes de atividades humanas que contenham radionuclídeos em quantidades superiores aos limites de eliminação especificados nas normas da Comissão Nacional de Energia Nuclear (CNEN) e para os quais a reutilização é imprópria ou não prevista;

- Grupo D: resíduos que não apresentem risco biológico, químico ou radiológico à saúde ou ao meio ambiente, podendo ser equiparados aos resíduos domiciliares;

- Grupo E: materiais perfurocortantes ou escarificantes.

Segundo Bartholomeu e Caixeta-Filho (2011), os RSS representam uma pequena parcela (de 1 a 3\%) dos resíduos sólidos urbanos (RSU). Entretanto, de acordo com a Resolução CONAMA nº 358/2005, devido às características específicas e aos riscos apresentados por esses resíduos, estes devem ser segregados na fonte de geração, segundo a classificação supracitada, e gerenciados de forma diferenciada.

A quantidade e natureza de RSS gerada diariamente nos diversos tipos de estabelecimentos de saúde ainda não é conhecida, pois não há dados gerais mensuráveis de geração diária. Além disso, diversos fatores influenciam na taxa de geração dos RSS, dentre eles: tipos de atividades de assistência adotada no estabelecimento, a quantidade de serviços de assistência realizados, o número de pacientes atendidos e de profissionais envolvidos, e até fatores sazonais. De acordo com Cussiol (2005), em pesquisa realizada na cidade de Belo Horizonte (MG), em hospital pediátrico, constatou-se que a geração média de resíduos encontrada foi de 3,17 kg.leito ${ }^{-1}$ ocupado por dia, sendo que 1,28 kg.leito ${ }^{-1}$ são os potencialmente infectantes, e 1,89 kg.leito ${ }^{-1}$, comuns. Porém, vários estabelecimentos de assistência à saúde, como unidades básicas de saúde (UBSs), consultórios dentários, farmácias, dentre outros, não realizam serviços de internação de pacientes, tornando difícil estabelecer uma relação simples para estimar a taxa de geração dos RSS.

De acordo com a Resolução CONAMA n 358/2005, a responsabilidade do gerenciamento dos RSS cabe ao gerador, desde a geração até a sua disposição final. Na prática, no Estado, observa-se que esse gerenciamento é descontínuo e com poucas ações, resultando apenas em pequenas melhorias no cenário geral, para a gestão dos RSS.

Nesse contexto, o presente trabalho teve como objetivo realizar o diagnóstico da aplicabilidade da Resolução CONAMA n 358/2005 e da RDC n 306/2004 em relação ao gerenciamento dos RSS nos estabelecimentos públicos de serviços de saúde, em 48 municípios da região metropolitana e central de Minas Gerais, contemplados com o recebimento do Imposto sobre Circulação de Mercadorias e Serviços (ICMS) ecológico.

\section{ÁREA DE ESTUDO}

O estudo foi realizado em 48 municípios localizados na região central, situados a uma área de abrangência da região de $276 \mathrm{~km}$ da capital, Belo Horizonte, do Estado de Minas Gerais. A população total (urbana e rural) do conjunto pesquisado foi de 1.701.510 habitantes (IBGE, 2010).

Os municípios pesquisados foram: Araçaí, Barão de Cocais, Belo Vale, Bom Jesus do Amparo, Brumadinho, Cachoeira da Prata, Caeté, Capim Branco, Caranaíba, Casa Grande, Catas Altas, Confins, Contagem, Cordisburgo, Corinto, Cristiano Otoni, Curvelo, Entre Rios de Minas, Florestal, Ibirité, Igarapé, Itabirito, Lagoa Santa, Mário Campos, Matozinhos, Monjolos, Morro da Garça, Nova Lima, Papagaios, Paraopeba, Pedro Leopoldo, Piedade dos Gerais, Prudente de Morais, Queluzita, Raposos, Rio Acima, Santana do Pirapama, Santana do Riacho, Santana dos Montes, Santo Hipólito, São Brás do Suaçuí, São Gonçalo do Rio Abaixo, São Joaquim de Bicas, São José da Lapa, Sarzedo, Sete Lagoas, Três Marias, e Vespasiano. 
Todos os municípios objetos do estudo são contemplados com recebimento do ICMS ecológico. Ressalta-se que foram contemplados na pesquisa somente estabelecimentos públicos, prestadores de serviços de assistência à saúde nessas localidades.

Para que o município seja habilitado no critério ecológico da lei, é imprescindível que adote sistemas de tratamento regularizados ambientalmente, para disposição de RSU e ou sistemas de esgotamento sanitário. Os municípios mineiros que adotam sistemas adequados de disposição de RSU recebem, como forma de incentivo, uma parcela da arrecadação do imposto sobre operações relativas à circulação de mercadorias e sobre prestações de serviços de transporte interestadual e intermunicipal e de comunicação (ICMS), o denominado ICMS ecológico subcritério saneamento ambiental (SEMAD, 2011).

Segundo a PNRS (Lei 12.305/2010), é de responsabilidade dos governos municipais a coleta e disposição final dos RSU, de forma a atender aos padrões de saúde pública e de qualidade ambiental. De acordo com a Fundação Estadual do Meio Ambiente (FEAM) (2012), todos os municípios objetos do estudo possuem um gerenciamento ambientalmente adequado para os RSU municipais, representados pelas cores marrom e verde na Figura 1.

Segundo o Panorama da Destinação dos Resíduos de Serviço de Saúde em Minas Gerais, realizado pela FEAM em 2011, pode-se observar que os RSS gerados nos municípios da região estudada são, em sua maioria, coletados e tratados via incineração por empresas privadas (FEAM, 2011).

\section{METODOLOGIA}

Para o levantamento da situação do gerenciamento dos RSS gerados nos estabelecimentos públicos de atenção à saúde, foram aplicados questionários enviados às Secretarias Municipais de Saúde das cidades pesquisadas, de agosto de 2012 a março de 2013, conforme Anexo I. A identificação dos estabelecimentos de saúde objetos da pesquisa foi

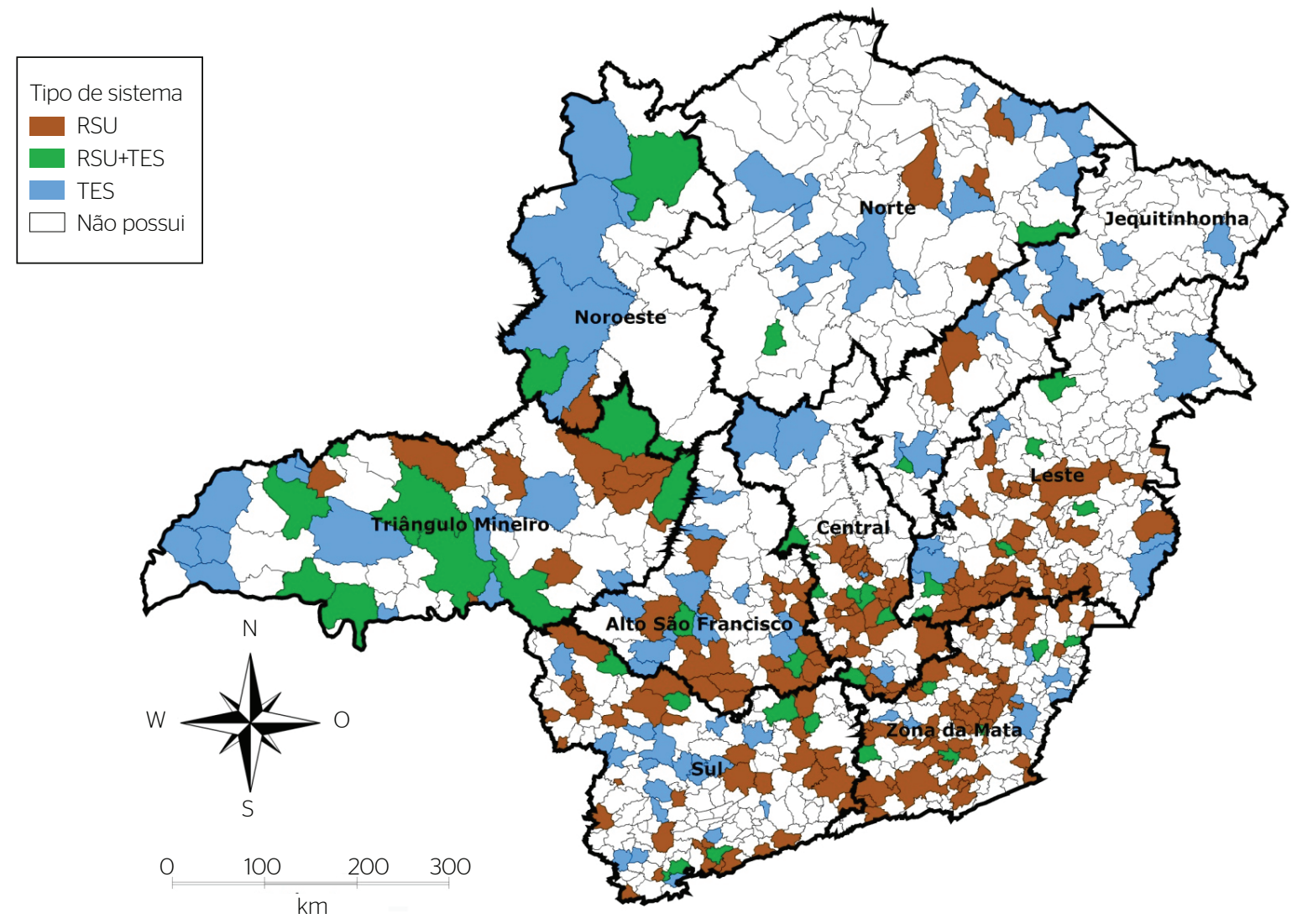

RSU: Resíduos Sólidos Urbanos; TES: Tratamento de Esgoto Sanitário

Fonte: Fundação Estadual do Meio Ambiente (FEAM). Relatório Executivo do Cadastro do ICMS Ecológico Subcritério Saneamento Ambiental 2012. Belo Horizonte: 2012.

Figura 1 - Municípios com sistemas de saneamento ambiental. 
realizada de acordo com o Cadastro Nacional de Estabelecimentos de Saúde (CNES), que é um banco de dados do Departamento de Informática do Sistema Único de Saúde (DATASUS) sobre a rede assistencial de serviços de saúde existente no país.

Para avaliação das respostas, as tipologias de atendimento à saúde foram agrupadas em quatro tipos de estabelecimentos, identificados por meio do CNES, devido às similaridades de procedimentos realizados, sendo assim definidos:

- hospitais, abrangendo as tipologias de hospital geral e hospital especializado e pronto socorro: são unidades destinadas a prestar assistência sanitária (com ou sem especialidades médicas) à população, além das quatro especialidades básicas: clínica médica, clínica cirúrgica, clínica ginecológico-obstétrica e clínica pediátrica;

- unidades básicas, contemplando as tipologias unidade básica, postos de saúde, policlínica e unidade mista: são estabelecimentos com atendimento de baixa complexidade, caracterizados por um conjunto de ações de saúde, para realização de primeiro atendimento às urgências médicas, de forma programada, a uma população determinada;

- farmácias públicas;

- consultórios odontológicos.

Os questionários da pesquisa foram elaborados buscando-se avaliar as principais diretrizes da Resolução CONAMA n 358/2005 para o gerenciamento dos RSS. Foram feitos questionamentos com relação aos seguintes itens: quantidade de estabelecimentos públicos de saúde existentes no município; número de atendimentos realizados; existência do Plano de Gerenciamento de Resíduos de Serviços de Saúde (PGRSS); os aspectos operacionais de segregação, acondicionamento, armazenamento, transporte, tratamento e disposição final.

Para efeitos de análise, foram considerados somente os aspectos de elaboração de PGRSS, segregação, coleta externa, tratamento e disposição final, na avaliação dos procedimentos operacionais e gerenciais aplicados pelos estabelecimentos geradores de RSS.

A elaboração e aplicação dos questionários foram realizadas pela Fundação Israel Pinheiro (FIP), com aprovação da FEAM, por intermédio do envio de mensagem eletrônica, no período de agosto de 2012 a março de 2013, às Secretarias Municipais de Saúde das cidades pesquisadas. O preenchimento foi realizado pelos secretários(as) municipais de saúde ou por responsável da área indicado pela administração municipal. Em alguns municípios em que foi realizada visita técnica para apuração do ICMS ecológico foi realizado um preenchimento assistido, com apoio técnico dos analistas ambientais da FIP.

As informações obtidas nos questionários foram tabuladas em estrutura de banco de dados em planilha eletrônica, para análise e avaliação da situação do gerenciamento dos RSS informada pelos municípios objetos do estudo.
Para melhor análise dos resultados, os municípios foram agrupados em faixas de estratificação de população, considerando-se a população total (urbana e rural), conforme mostrado na Tabela 1. Essa divisão tem como principal objetivo estratificar o perfil das tipologias de serviços de assistência à saúde, distribuídas no conjunto de municípios pesquisados. Os municípios com população até 20 mil hab. apresentaram um perfil de assistência à saúde de baixa complexidade; os municípios com população entre 20 e 50 mil e 50 e 100 mil hab. apresentaram um perfil de assistência de baixa a média complexidade; já os municípios acima de 100 mil hab. apresentaram um perfil de assistência à saúde de média a alta complexidade.

\section{RESULTADOS E DISCUSSÃO}

De acordo com as informações prestadas pelas Secretarias Municipais de Saúde, nas 48 cidades pesquisadas, somam 643 estabelecimentos públicos de assistência à saúde, sendo a distribuição percentual das tipologias apresentada na Figura 2.

Tabela 1 - Estratificação da população dos municípios mineiros pesquisados no período de agosto de 2012 a março de 2013.

\begin{tabular}{l|c|c} 
Grupos de municipios & $\begin{array}{c}\text { Quantidade } \\
\text { de municipios }\end{array}$ & $\begin{array}{c}\text { População } \\
\text { correspondente }\end{array}$ \\
\hline Até 20 mil hab. & 29 & 203.709 \\
\hline 20 a 50 mil hab. & 12 & 364.401 \\
\hline 50 a 100 mil hab. & 4 & 266.477 \\
\hline Mais de 100 mil hab. & 3 & 866.923 \\
\hline TOTAL & 48 & 1.701 .510 \\
\hline
\end{tabular}

Fonte: IBGE, 2010.

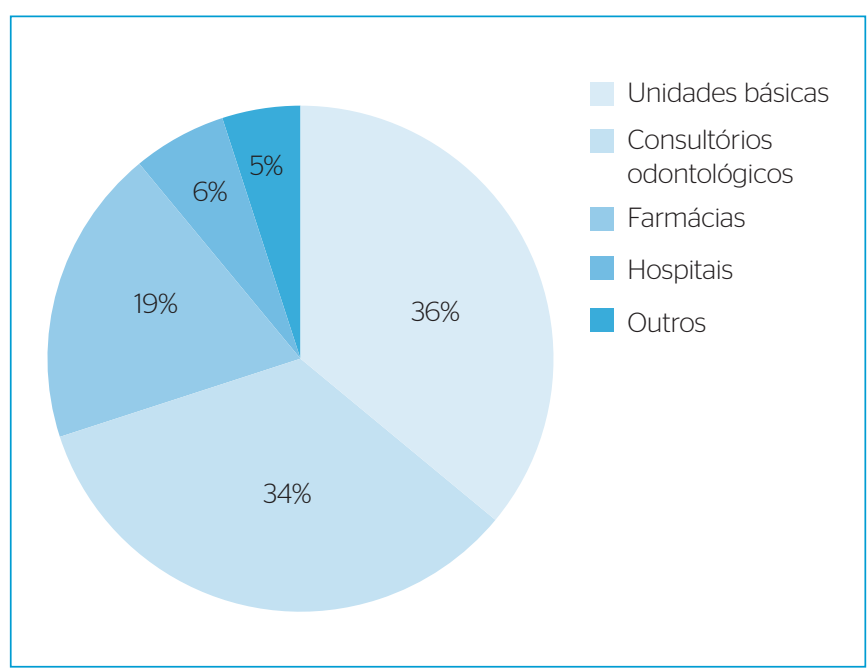

Figura 2 - Percentual de estabelecimentos públicos de saúde identificados nos municípios mineiros pesquisados no período de agosto de 2012 a março de 2014. 
No Estado de Minas Gerais, a competência para acompanhar a execução da elaboração e implantação dos PGRSSs nos estabelecimentos de assistência à saúde está a cargo da Vigilância Sanitária Estadual e dos municípios, sendo tal elaboração o primeiro aspecto avaliado. Também no art. 4 da Resolução CONAMA n 358/2005 é preconizada a obrigatoriedade da elaboração e implantação do PGRSS pelos estabelecimentos geradores de RSS.

Entretanto, verificou-se nas informações prestadas pelas Secretarias Municipais de Saúde das cidades pesquisadas que a maioria dos estabelecimentos públicos de saúde do município não elaborou o PGRSS (48\%), sendo que apenas $33 \%$ possuem o PGRSS para todas as unidades públicas de atendimento à saúde no município e 19\% possuem em apenas algumas unidades.

A ausência da elaboração e implantação do PGRSS reflete negativamente nas diversas fases do gerenciamento dos RSS, em especial nos seus princípios essenciais, que são a prevenção e a precaução. Outro aspecto que também tem uma repercussão negativa é que a falta de aplicação de critérios técnicos corretos de classificação, segregação e acondicionamento faz com que os resíduos misturados sejam considerados como os de maior periculosidade, o que acarreta destinação diferenciada e menos econômica. O planejamento (realizado a partir da elaboração do PGRSS) é ferramenta que pode diminuir gastos desnecessários na destinação dos RSS.

Outro fator importante observado é que, independentemente da faixa populacional, não houve atendimento, na íntegra, ao art. $4^{\circ} \mathrm{da}$ Resolução CONAMA nº 358/2005 pelos municípios em relação à elaboração do PGRSS. Observou-se que a obrigação estabelecida na resolução citada não foi cumprida. Portanto, a percepção da importância do planejamento, enquanto um processo eficiente de gestão, por parte desses geradores ainda não foi despertada nos participantes da pesquisa.

\section{Aspectos operacionais do gerenciamento dos resíduos de serviços de saúde}

\section{Segregação}

Para avaliar o aspecto "segregação dos RSS", foi realizado um cruzamento de dados entre a implantação de PGRSS e a informação de acondicionamento diferenciado. Assim, considerou-se ser impossível realizar a correta segregação dos RSS sem um planejamento dos aspectos de gerenciamento, assinalados no PGRSS, de forma que foi considerada correta a segregação dos resíduos na fonte apenas para os municípios que afirmaram possuir PGRSS em todas as unidades, ou seja, em apenas 33\% do conjunto pesquisado. A Figura 3 mostra o aspecto "Segregação dos RSS" comparado ao aspecto "Existência de PGRSS".

Embora seja obrigatória a segregação de todas as classes de resíduos, preconizada pelo art. 14 da Resolução CONAMA no 358/2005, percebeu-se que o acondicionamento diferenciado dos RSS nos municípios pesquisados ocorre com maior relevância em relação ao grupo de resíduos biológicos (Grupo A) e perfurocortantes (Grupo E). Isso porque foi informada a utilização apenas de sacos brancos específicos (que se destinam aos resíduos biológicos) e caixas para perfurocortantes.

Outro fato relevante observado é que os resíduos com risco químico (Grupo B) gerados nesses estabelecimentos sequer foram mencionados na pesquisa, o que sinaliza não têm recebido a atenção necessária, sendo, provavelmente, descartados sem a devida segregação. Esses resíduos, segundo a Resolução CONAMA no 358/2005, podem ser perigosos por possuir característica química de inflamabilidade, corrosividade,

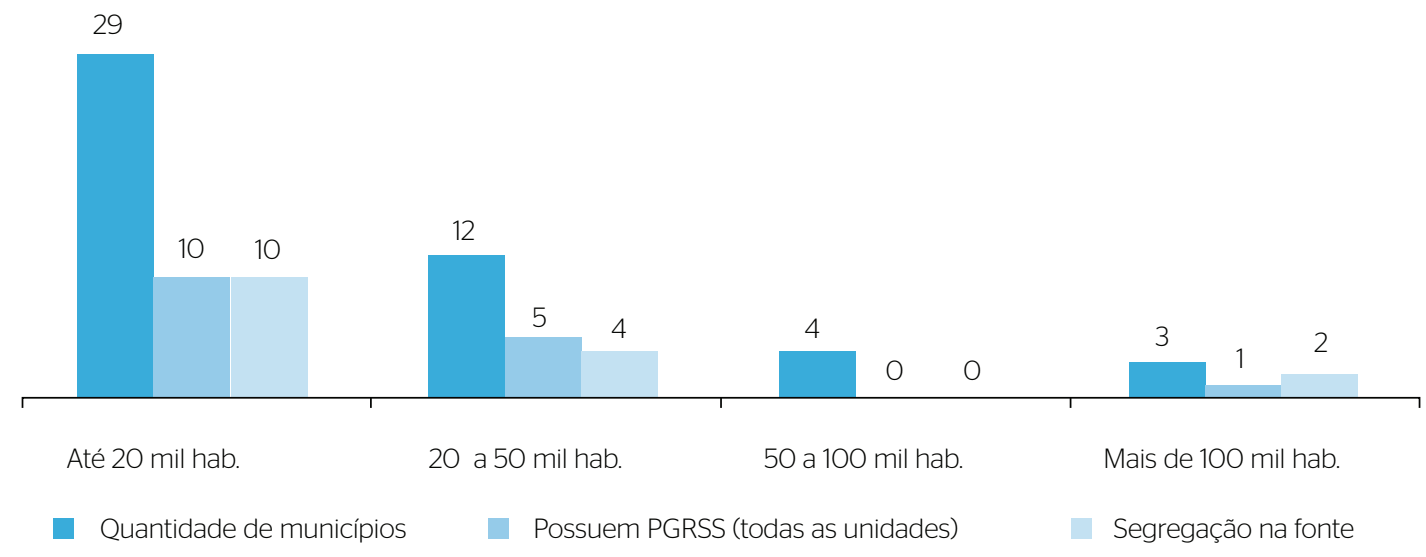

PGRSS: Plano de Gerenciamento de Resíduos de Serviços de Saúde.

Figura 3 - Atendimento ao art. 4 e ao art.14 da Resolução CONAMA no 358/2005 pelos municípios pesquisados - elaboração de Plano de Gerenciamento de Resíduos de Serviços de Saúde e segregação na fonte. 
reatividade ou toxicidade, conforme definido na NBR 10.004/2004, da $\mathrm{ABNT}$, e devem ser submetidos a acondicionamento, armazenamento, coleta, tratamento e disposição final específicos. Dentre os resíduos com risco químico, podem-se citar os produtos hormonais, antimicrobianos, quimioterápicos, antineoplásicos e citostáticos, resíduos contendo metais pesados, alguns tipos de reagentes de laboratório, dentre outros.

A inexistência do gerenciamento dos resíduos químicos, detectada na pesquisa, demonstra um cenário preocupante. A região do estudo apresenta uma característica de maior concentração dos serviços públicos de assistência à saúde de alta complexidade, realizados em todo o Estado de Minas Gerais, definida pela Secretaria Estadual de Saúde de Minas Gerais (SESMG) como Macrorregional Central de Saúde 1. Assim, possivelmente a geração dos resíduos com risco químico tende a ser maior nesta do que em outras regiões do Estado e, no entanto, eles sequer foram mencionados. Ressalta-se que em municípios com até 20 mil hab. a geração de resíduos químicos é quase inexistente, devido a não realização de serviços assistência à saúde de alta complexidade.

Observou-se também que $82 \%$ dos municípios analisados informaram descartar de forma diferenciada os resíduos comuns (Grupo D) gerados nesses estabelecimentos. Entretanto, nenhuma Secretaria de Saúde dos municípios pesquisados respondeu realizar coleta seletiva e encaminhamento para reciclagem dos materiais recicláveis não contaminados gerados nessas unidades, como papel, papelão, plástico e metal. Embora não se possa afirmar, tudo indica que, ao contrário do informado, a maioria desses resíduos deve ser coletada com os demais resíduos de saúde do estabelecimento.

\section{Quantificação dos resíduos}

A quantificação dos resíduos foi um item que as Secretarias de Saúde Municipais tiveram muita dificuldade de responder: $14,5 \%$ informaram não quantificar os resíduos de saúde gerados, deixando o item em branco, ou preenchendo com alguma denominação, como "Sem estimativa".

Ademais, não foi percebido critério técnico na utilização de unidades de medida, sendo que cada representante do município respondeu de acordo com a sua percepção, embora tenha sido assinalada no questionário a unidade de medida para quantificação. Além disso, não foi informada a geração por grupo de classificação dos RSS, tampouco foi informada a tipologia gerada de cada estabelecimento de saúde instalado no município.

Considerando os valores apresentados, padronizando as unidades fornecidas e considerando nulos os valores que não foram informados, somam-se 4,81 t.dia-1 de RSS para todos os municípios pesquisados. Essas constatações não podem ser consideradas como valores reais, de geração, uma vez que foi percebido baixo índice de conhecimento em relação ao gerenciamento dos RSS, ressaltando-se, mais uma vez, que apenas 33\% do conjunto pesquisado afirmaram possuir PGRSS elaborado para todos os estabelecimentos de saúde.

\section{Coleta, transporte externo e destinação final}

A coleta dos RSS, na grande maioria dos municípios, é realizada de forma diferenciada por empresas privadas. Dos 48 municípios pesquisados, apenas 7 não realizam a coleta dos RSS de forma inadequada ambientalmente.

Atualmente, no Estado de Minas Gerais, a coleta e o transporte externo dos RSS são licenciados ambientalmente de acordo com as exigências legais e as Normas da ABNT-NBR12810 (1993). Dentro do conjunto formado pela estratificação da população, o grupo com a população até 20 mil hab. foi o que apresentou maior número de municípios que não realizam coleta adequada, conforme apresentado na Figura 4.

Quanto ao tratamento e à disposição final, a Resolução CONAMA $n^{\circ} 358 / 2005$ estabelece que alguns tipos de resíduos devem ser tratados ou destinados de formas diferenciadas, de acordo com a sua classificação. Conforme o informado nos questionários, os RSS acondicionados em sacos brancos (e, portanto, considerados com risco biológico) são destinados, nos municípios pesquisados, da seguinte forma: $4 \%$ dos municípios encaminham para autoclave; $86 \%$, para unidades de incineração; $\mathrm{e}$ $10 \%$, para disposição sem tratamento. Não foi explicitado se os resíduos dispostos sem tratamento pertencem ao subgrupo A4, que são os que não precisam ser tratados, nem aos subgrupos do Grupo A, que são os encaminhados para autoclavagem ou incineração. Portanto, há indicativos de que todo o Grupo A é encaminhado para tratamento, sem segregação em seus subgrupos, o que onera o serviço de saúde sem necessidade.

Não foi mencionada, nos questionários, a destinação dada aos RSS pertencentes aos demais grupos. Apesar de a Resolução CONAMA $n^{\circ}$ 358/2005 e a RDC ANVISA no 306/2004 preconizarem que determinados grupos de RSS necessitam de tratamento e destinação de acordo com o grupo ao qual pertence o RSS, pode-se perceber, diante da realidade detectada por meio dos questionários da pesquisa, que essas diretrizes não estão sendo cumpridas pelos geradores de RSS na área de estudo em questão.

Observou-se ainda que os municípios pesquisados, de forma geral, optaram por destinar os RSS gerados de forma diferenciada, encaminhando a maioria para unidades de incineração de RSS e autoclaves, mesmo não cumprindo outras diretrizes da Resolução CONAMA no 358/2005, tais como: elaboração e implantação do PGRSS, obrigatoriedade de segregação na fonte de geração. Isso pode ser devido ao fato de os agentes públicos municipais, por receberem o ICMS ecológico, terem atentado para a questão da destinação final adequada também para os RSS.

Ressalta-se que a área de estudo é uma das maiores áreas de geração de RSS no Estado de Minas Gerais, devido aos princípios de regionalização e hierarquização na organização das redes de atenção terciária à saúde, conforme estabelecido no Plano Diretor de Regionalização (PDR), elaborado por Malachias, Leles e Pinto (2011) em parceria com a Secretaria de Estado de Saúde de Minas Gerais (SESMG). A região concentra também o maior número de instalações de tecnologias para tratamento de RSS, conforme dados publicados pela FEAM em 2011, 
tendo como modalidades de tratamento as tipologias de autoclave e incineração e como disposição final os aterros sanitários e Classe I.

\section{Resultados comparativos}

Após a análise de cada aspecto do gerenciamento dos RSS separadamente, pode-se observar na Figura 5 que os aspectos internos aos estabelecimentos de atendimento à saúde (elaboração e implantação do PGRSS e segregação dos resíduos na fonte) são os mais preocupantes, uma vez que a maioria $(66,7 \%)$ dos municípios encontra-se em desacordo com a Resolução CONAMA nº 358/2005.

Já os aspectos externos de gerenciamento dos RSS avaliados (coleta externa e destinação final) estão sendo cumpridos pela maioria $(87,5 \%)$ dos municípios. Esse avanço talvez possa ser explicado por duas variáveis: o recebimento do ICMS pelos municípios e a proximidade dos municípios com a área com maior incidência de instalação de tecnologias (incineração e autoclave) para tratamento de RSS no Estado de Minas Gerais.

Entretanto, como a segregação na fonte é deficitária, pode-se inferir que essa destinação final realizada não é satisfatória, uma vez que do total de resíduos coletados, grande parte não precisaria receber tratamento, por serem resíduos comuns coletados com os que necessitam de tratamento, elevando o percentual de geração nesses estabelecimentos de resíduos perigosos.

Na Figura 5 é apresentado o índice de cumprimento dos aspectos de gerenciamento dos RSS avaliados.

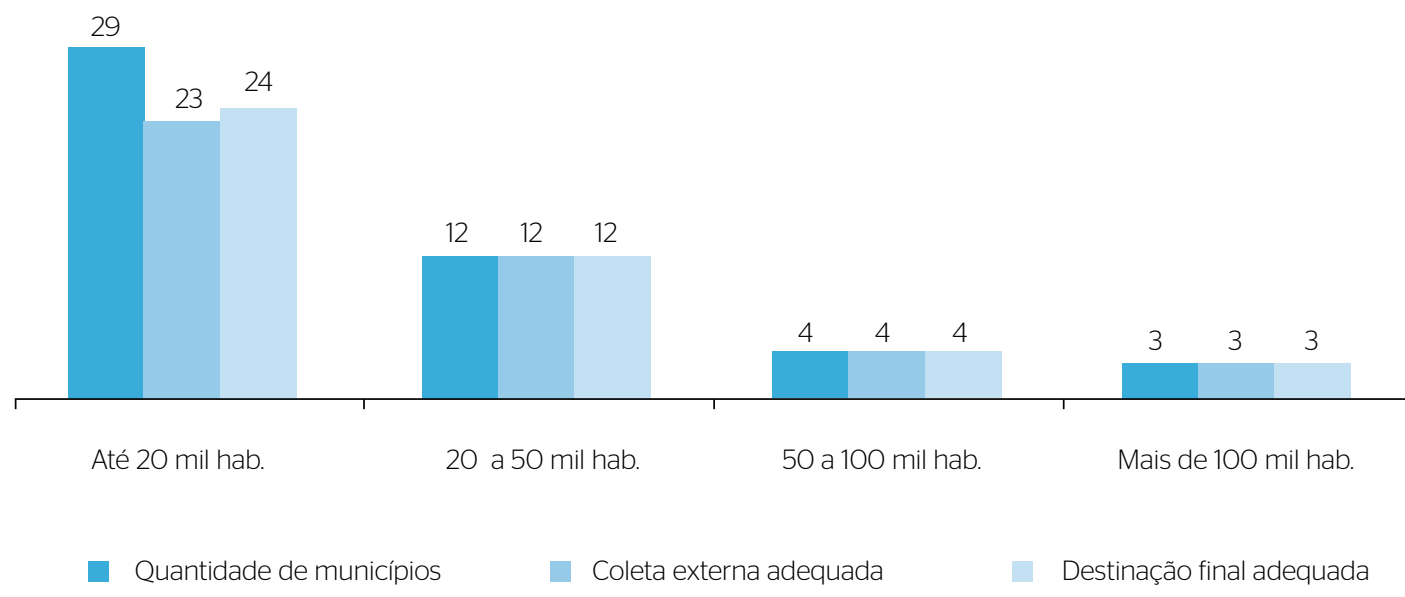

Figura 4 - Atendimento ao art. 8 da Resolução CONAMA n 358/2005 e à Norma da ABNT para coleta e transporte dos resíduos de serviços de saúde.

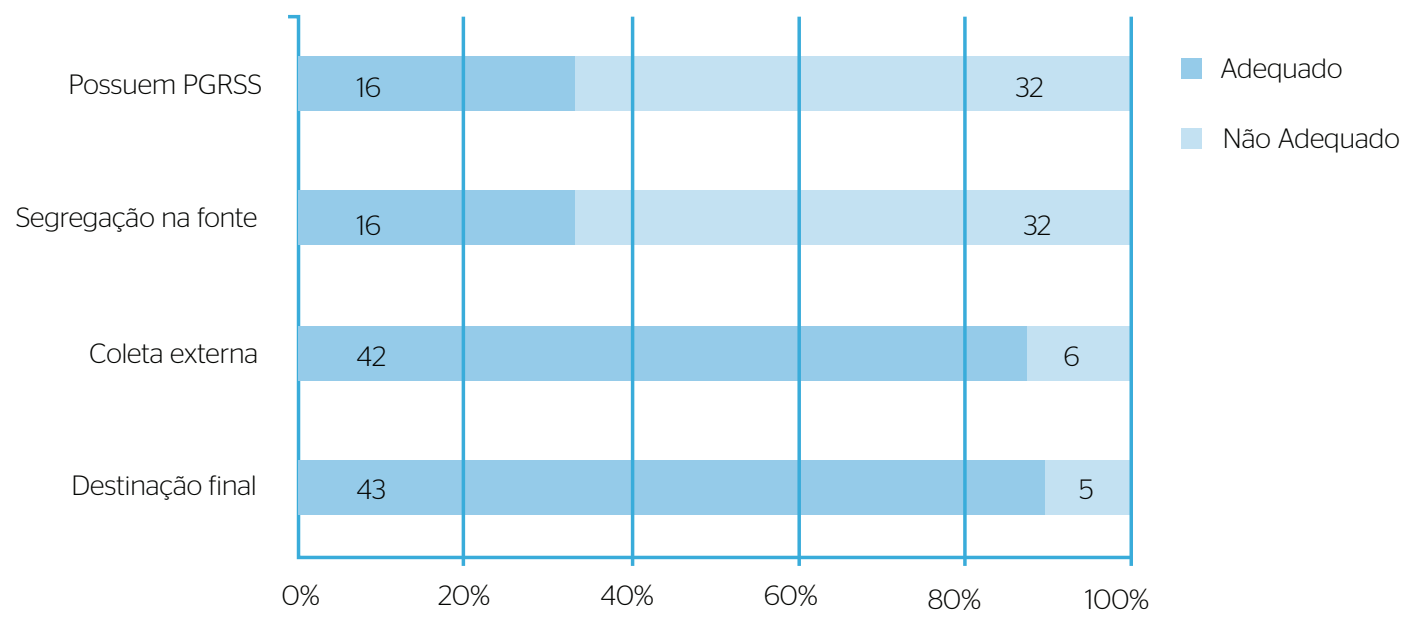

PGRSS: Plano de Gerenciamento de Resíduos de Serviços de Saúde.

Figura 5 - Atendimento aos aspectos de gerenciamento de resíduos de serviços de saúde em municípios mineiros pesquisados no período de agosto de 2013 a março de 2014. 


\section{CONSIDERAÇÕES FINAIS}

Observou-se, a partir da análise dos questionários respondidos pelas Secretarias de Saúde Municipais pesquisadas, que poucas ações vêm sendo realizadas em relação ao gerenciamento dos RSS. Foram percebidas como ações adequadas, em cumprimento à Resolução CONAMA n 358/2005: o acondicionamento diferenciado adequado para os Grupos A e E em grande parte dos municípios pesquisados; e a coleta e a destinação final diferenciada em $90 \%$ dos municípios que destinam seus resíduos para autoclavar e incinerar. Entretanto, pouca atenção é dada aos demais aspectos de gerenciamento e aos outros grupos de classificação dos RSS.

As principais dificuldades no âmbito do gerenciamento dos RSS nos estabelecimentos públicos de saúde dos municípios estudados encontram-se na fase intraestabelecimento, ou seja, na elaboração e implantação do PGRSS e na segregação dos resíduos na fonte. De acordo com os questionários avaliados, apenas 33\% dos municípios afirmaram possuir PGRSS e realizar a segregação na fonte de geração, embora a Resolução CONAMA n 358/2005 afirme a obrigatoriedade desses aspectos como essenciais ao gerenciamento dos RSS.

Conclui-se, então, que o gerenciamento clássico (da década de 1990) dos RSS ainda é vigente, ou seja, todos os resíduos gerados no estabelecimento de saúde são considerados perigosos e infectantes, o que pode ser considerada uma negligência perante as políticas públicas de gestão de resíduos sólidos. Assim sendo, é muito provável que resíduos sem características primárias de periculosidade estejam sendo encaminhados para tratamento diferenciado (incineração e autoclavagem), o que não seria necessário. A proposta final é a de fomentar nos serviços de atendimento à saúde a implantação de uma gestão ambientalmente efetiva dos resíduos que geram, a fim de utilizar melhor os recursos públicos investidos nessa área.

\section{REFERÊNCIAS}

ANVISA - AGÊNCIA NACIONAL DE VIGILÂNCIA SANITARIA. (2004) Resolução da Diretoria Colegiada - RDC no 306, de 7 de dezembro de 2004. Dispõe sobre o Regulamento Técnico para o gerenciamento de resíduos de serviços de saúde. Publicada no DOU, de 10 de dezembro de 2004. Brasília: ANVISA.

ABNT - ASSOCIAÇÃO BRASILEIRA DE NORMAS TÉCNICAS. (2004) NBR 10.004. Resíduos sólidos: classificação. Rio de Janeiro: ABNT.

ABNT - ASSOCIAÇÃO BRASILEIRA DE NORMAS TÉCNICAS. (1993) NBR 12.807. Resíduos de serviços de saúde: terminologia. Rio de Janeiro: ABNT.

ABNT - ASSOCIAÇÃO BRASILEIRA DE NORMAS TÉCNICAS. (1993) NBR 12.808. Resíduos de serviços de saúde: classificação. Rio de Janeiro: ABNT.

ABNT - ASSOCIAÇÃO BRASILEIRA DE NORMAS TÉCNICAS. (1993) NBR 12.809. Manuseio de resíduos de serviços de saúde: procedimento. Rio de Janeiro: ABNT.

ABNT - ASSOCIAÇÃO BRASILEIRA DE NORMAS TÉCNICAS. (1993) NBR 12.810. Coleta de resíduos de serviços de saúde: procedimento. Rio de Janeiro: ABNT.

BARTHOLOMEU, D.B. \& CAIXETA-FILHO, J.V. (Org.). (2011) Logística ambiental de resíduos sólidos. São Paulo: Atlas.

BRASIL. Lei no 12.305, de 2 de agosto de 2010. (2010) Institui a Política Nacional de Resíduos Sólidos; altera a Lei no 9.605, de 12 de fevereiro de 1998; e dá outras providências. Publicação no DOU, de 03 de agosto de 2010 .

BRASIL. Ministério do Meio Ambiente. Conselho Nacional do Meio Ambiente - CONAMA. (2005) Resolução no 358, de 29 de abril de 2005. Dispõe sobre o tratamento e a disposição final dos resíduos dos serviços de saúde e dá outras providências. Publicada no DOU no 84 , de 4 de maio de 2005, Seção 1, p. 63-65.
BRASIL. Ministério da Saúde. Secretaria Executiva - Projeto REFORSUS. (2001) Manual de Gerenciamento de Resíduos de Serviços de Saúde. Disponível em: <http://bvsms.saude.gov.br/bvs/publicacoes/Manual_ RSS_Parte1.pdf>. Acesso em: 10 fev. 2014.

CUSSIOL, N.A.M. (2005) Disposição final de resíduos potencialmente infectantes de serviços de saúde em célula especial e por co-disposição com resíduos sólidos urbanos. Tese (Doutorado em Saneamento, Meio Ambiente e Recursos Hídricos) - Universidade Federal de Minas Gerais, Belo Horizonte.

FEAM - FUNDAÇÃO ESTADUAL DO MEIO AMBIENTE. (2011) Panorama da destinação de Resíduos de Serviço de Saúde em Minas Gerais. Publicação na página eletrônica da FEAM. Disponível em: <http://www. feam.br/minas-rss-destinacao-sustentavel>. Acesso em: 27 jan. 2014

FEAM - FUNDAÇÃO ESTADUAL DO MEIO AMBIENTE. (2012) Situação do tratamento e disposição final dos RSU de Minas Gerais em 2012. Belo Horizonte. Disponível em: <http://www.feam.br/minas-sem-lixoes>. Acesso em: 25 jan. 2014.

IBGE - INSTITUTO BRASILEIRO DE GEOGRAFIA E ESTATISTICA. (2010) Banco de dados SIDRA, Censo demográfico de 2010: Universo Características da População e dos Domicilios. Disponível em: <http://www. sidra.ibge.gov.br/cd/cd2O1ORgaAdAgsn.asp>. Acesso em: 26 mar. 2014.

MALACHIAS, I.; LELES, F.A.G.; PINTO, M.A.S. (2011) Plano Diretor de Regionalização da Saúde de Minas Gerais (PDR/MG). Belo Horizonte, março de 2011. Disponível em: <http://www.saude.mg.gov.br/images/ documentos/Livro\%2OPlano\%20Diretor\%20de\%20Regionalizacao\%20 -\%2Oultima\%2Oversao.pdf>. Acesso em: 10 fev. 2014.

SEMAD - SECRETARIA DE ESTADO DE MEIO AMBIENTE E DESENVOLVIMENTO SUSTENTÁVEL. ICMS-Ecológico. (2011) Publicação na página eletrônica da SEMAD, 2011. Disponível em: <http://www. meioambiente.mg.gov.br/icms-ecologico>. Acesso em: 10 fev. 2014. 
ANEXO I

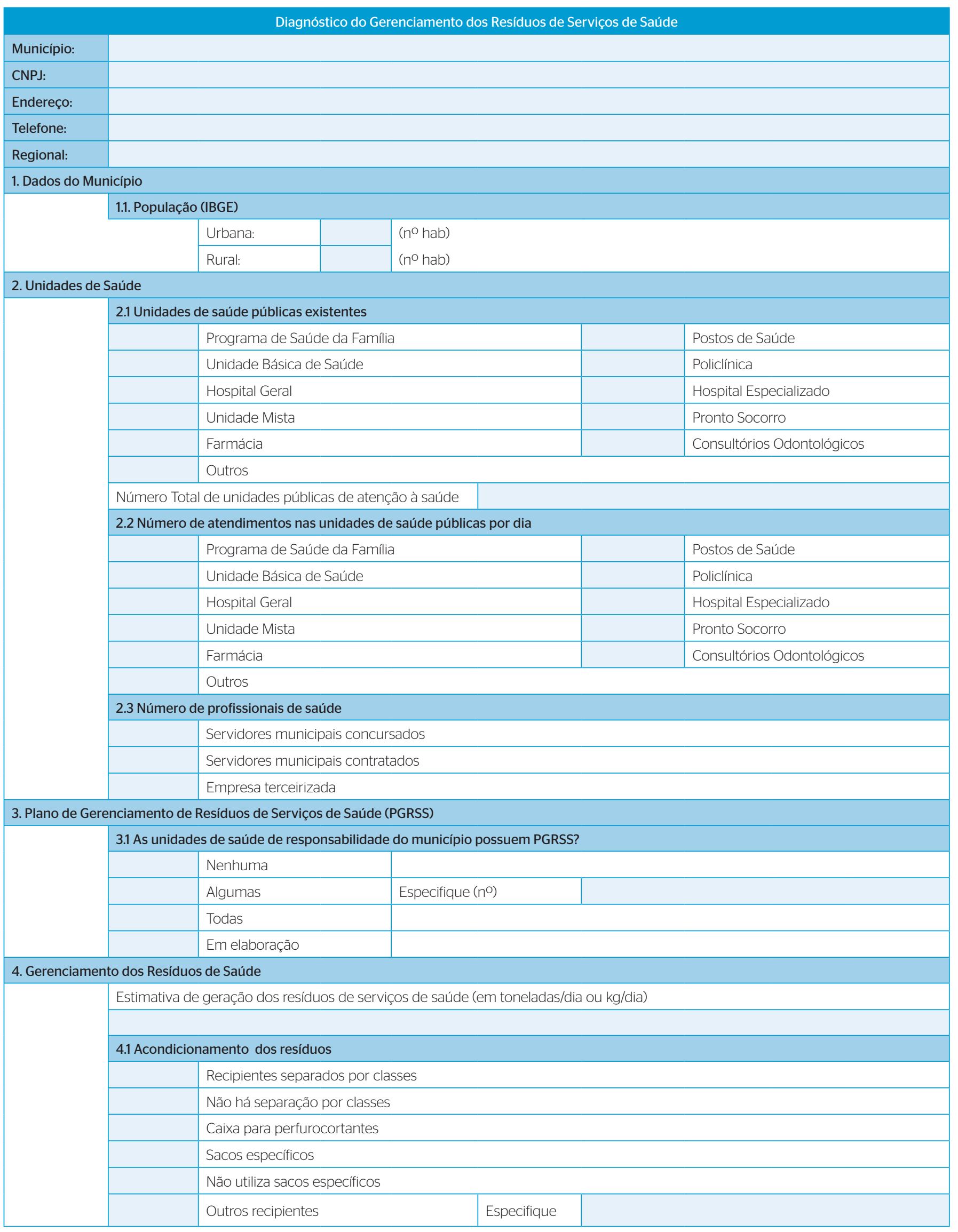


ANEXO I - Continuação.

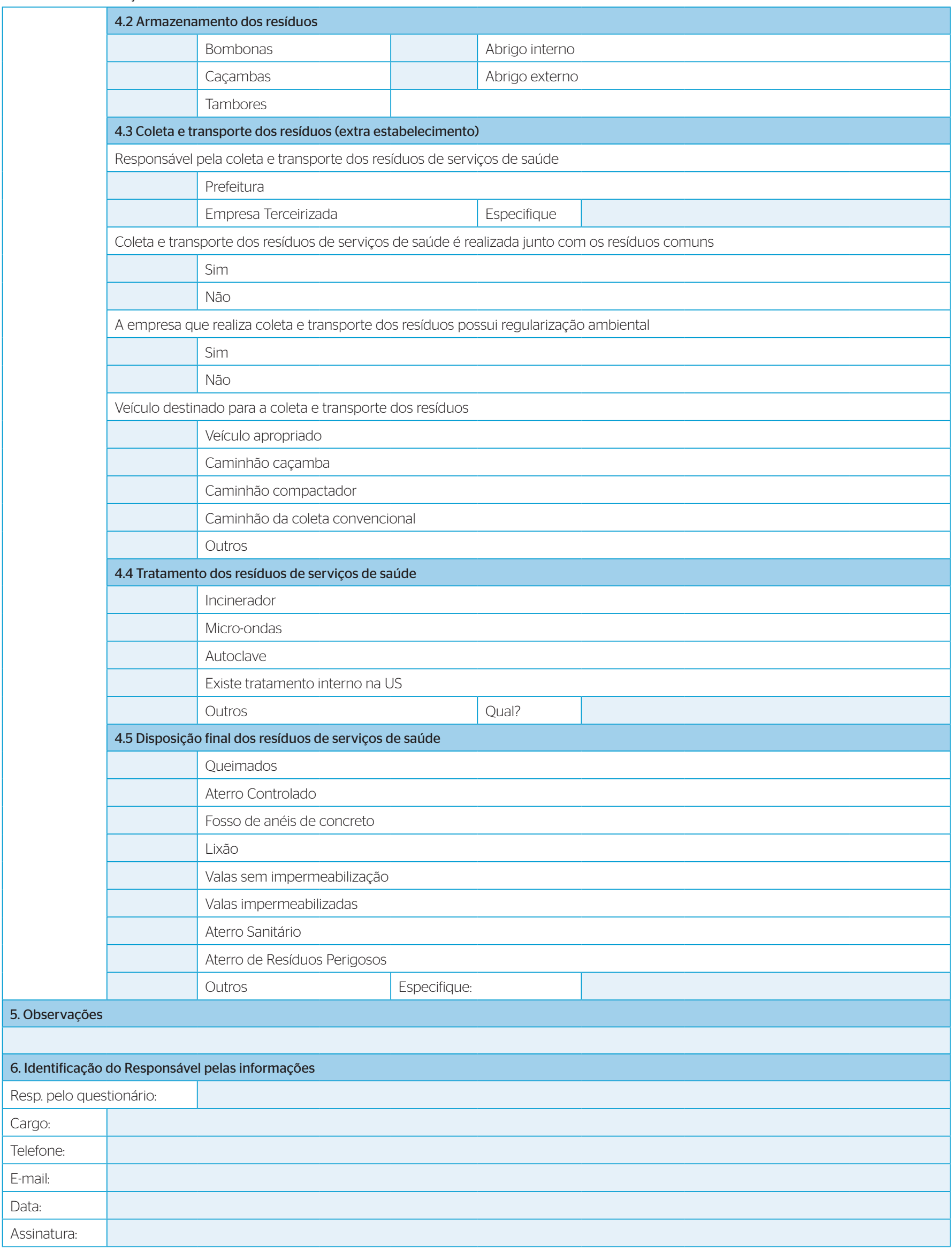

